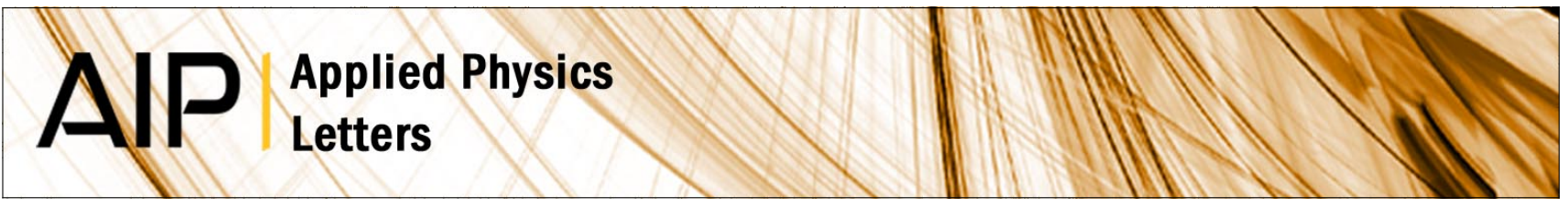

\title{
Liquid crystal based nonlinear fishnet metamaterials
}

Alexander Minovich, James Farnell, Dragomir N. Neshev, lan McKerracher, Fouad Karouta et al.

Citation: Appl. Phys. Lett. 100, 121113 (2012); doi: 10.1063/1.3695165

View online: http://dx.doi.org/10.1063/1.3695165

View Table of Contents: http://apl.aip.org/resource/1/APPLAB/v100/i12

Published by the American Institute of Physics.

\section{Related Articles}

Image acceleration in parallel magnetic resonance imaging by means of metamaterial magnetoinductive lenses AlP Advances 2, 022136 (2012)

Inhomogeneous nanostructured honeycomb optical media for enhanced cathodo- and under-x-ray luminescence J. Appl. Phys. 111, 103101 (2012)

Broadband Purcell effect: Radiative decay engineering with metamaterials

Appl. Phys. Lett. 100, 181105 (2012)

Switchable hyperbolic metamaterials with magnetic control

Appl. Phys. Lett. 100, 161108 (2012)

An origami tunable metamaterial

J. Appl. Phys. 111, 084905 (2012)

\section{Additional information on Appl. Phys. Lett.}

Journal Homepage: http://apl.aip.org/

Journal Information: http://apl.aip.org/about/about_the_journal

Top downloads: http://apl.aip.org/features/most_downloaded

Information for Authors: http://apl.aip.org/authors

\section{ADVERTISEMENT}
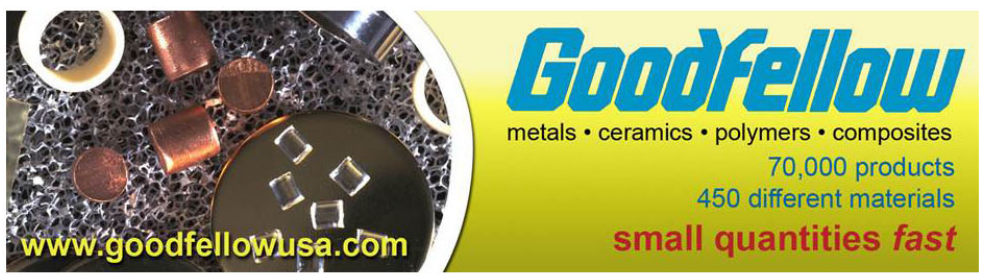


\title{
Liquid crystal based nonlinear fishnet metamaterials
}

\author{
Alexander Minovich, ${ }^{1}$ James Farnell, ${ }^{1}$ Dragomir N. Neshev, ${ }^{1, a)}$ Ian McKerracher, ${ }^{2}$ \\ Fouad Karouta, ${ }^{3}$ Jie Tian, ${ }^{3}$ David A. Powell, ${ }^{1}$ Ilya V. Shadrivov, ${ }^{1}$ Hark Hoe Tan, ${ }^{2}$ \\ Chennupati Jagadish, ${ }^{2}$ and Yuri S. Kivshar ${ }^{1}$ \\ ${ }^{1}$ Nonlinear Physics Centre, Centre for Ultrahigh-bandwidth Devices for Optical Systems (CUDOS), Research \\ School of Physics and Engineering, The Australian National University, Canberra ACT 0200, Australia \\ ${ }^{2}$ Electronic Materials Engineering, Research School of Physics and Engineering, The Australian National \\ University, Canberra ACT 0200, Australia \\ ${ }^{3}$ Australian National Fabrication Facility ACT Node, Research School of Physics and Engineering, \\ The Australian National University, Canberra ACT 0200, Australia
}

(Received 9 January 2012; accepted 29 February 2012; published online 21 March 2012)

\begin{abstract}
We study experimentally the nonlinear properties of fishnet metamaterials infiltrated with nematic liquid crystals and find that moderate laser powers result in significant changes of the optical transmission of the composite structures. We also show that the nonlinear response of our structure can be further tuned with a bias electric field, enabling the realization of electrically tunable nonlinear metamaterials. @ 2012 American Institute of Physics. [http://dx.doi.org/10.1063/1.3695165]
\end{abstract}

Many applications of photonic structures require the ability to change their properties all-optically through a nonlinear change of their refractive index. However, in natural materials, a sizable optical nonlinearity requires the use of ultra-high light intensities and powers, hindering the practical use of optical nonlinearities. An important approach for the enhancement of nonlinearity is offered by the significant field concentration and subwavelength confinement in plasmonic and metamaterial structures, employing the increased density of electromagnetic states near a metal surface. ${ }^{1,2}$ Especially, important is the case of field enhancement in metamaterial structures, because this can influence both their electric and magnetic nonlinear response. ${ }^{3-6}$ As such the development of switchable and nonlinear metamaterials has been identified as a key area in the field of metamaterials. ${ }^{7}$ Indeed, a number of theoretical proposals have revealed that a range of new nonlinear phenomena are possible in metamaterials, including enhancement of nonlinearity, backwardwave phase matching for harmonic generation, and parametric amplification (see Refs. 8-10 and references therein). The experimental verification of such metamaterial-specific ideas is still lacking, with the exception of enhanced harmonic generation. ${ }^{11-13}$

One of the major difficulties in such experimental verification is that the thickness of currently available optical metamaterials is of the order of the optical wavelength or smaller. Therefore, the nonlinear change in refractive index must be very large to control the transmission response with incident light power. Achieving such a strong nonlinear shift in a thin layer poses a significant challenge to the experimental studies of nonlinear metamaterials, with only a few examples having been reported for plasmonic structures. ${ }^{14,15}$

An important proof for the feasibility of the enhanced nonlinearity in metamaterials can be achieved by embedding in their structure dielectrics with a giant nonlinear optical response such as liquid crystals (LCs). ${ }^{16}$ In this way, the

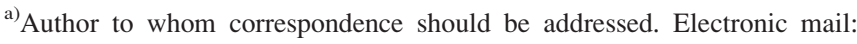
Dragomir.Neshev@anu.edu.au.
}

giant response of the dielectric can compensate for the subwavelength thickness of the materials. Indeed the large change of refractive index of the nematic LC due to molecular re-orientation caused by external electric field has been utilized to control the transmission through LC infiltrated photonic ${ }^{17}$ and plasmonic structures. ${ }^{18,19}$ Liquid-crystal tunability of magnetic and negative index metamaterials has also been suggested ${ }^{20-22}$ and experimentally verified for thermal tunability of purely magnetic metamaterials. ${ }^{23}$ Nevertheless, the nonlinear response of negative index optical metamaterials has not been reported to date.

In this letter, we study experimentally the nonlinear optical response of double fishnet metamaterials structures infiltrated with a nematic LC. We show that indeed the LC infiltration provides an efficient mechanism for all-optical tunability of the fishnet structures operating at optical frequencies. We show that moderate laser powers can influence the transmission through the fishnets, being further tunable by the external electric field.

The fishnet metamaterials are fabricated on a glass substrate using gold and $\mathrm{MgF}_{2}$ as metal and dielectric layers, respectively, as schematically shown in Fig. 1(a). For nanostructuring of the metal-dielectric layers, we use focused ion beam milling, to create a fishnet structure as shown in the scanning electron micrograph in Fig. 1(b). ${ }^{24}$ The metamaterial parameters are chosen to achieve effective negative index of the fishnets in the telecommunication spectral range and are optimized for maximum sensitivity of the optical transmission to a change in the refractive index of the LC inside the holes. ${ }^{22}$ This fishnet metamaterial is then infiltrated with E7 nematic LC and sandwiched on the top with a cover-glass slide coated with a transparent indium tin oxide (ITO) electrode and a polyvinyl alcohol (PVA) layer [Fig. 1(c)]. Micro-trenches are formed in the PVA layer in order to facilitate unidirectional horizontal pre-alignment of the LC molecules. In addition, a low-frequency AC voltage $(1 \mathrm{kHz}, \leq 100 \mathrm{~V})$ can be applied between the top ITO electrode and the bottom gold film for electric control of the LC molecular alignment [Fig. 1(c)]. 


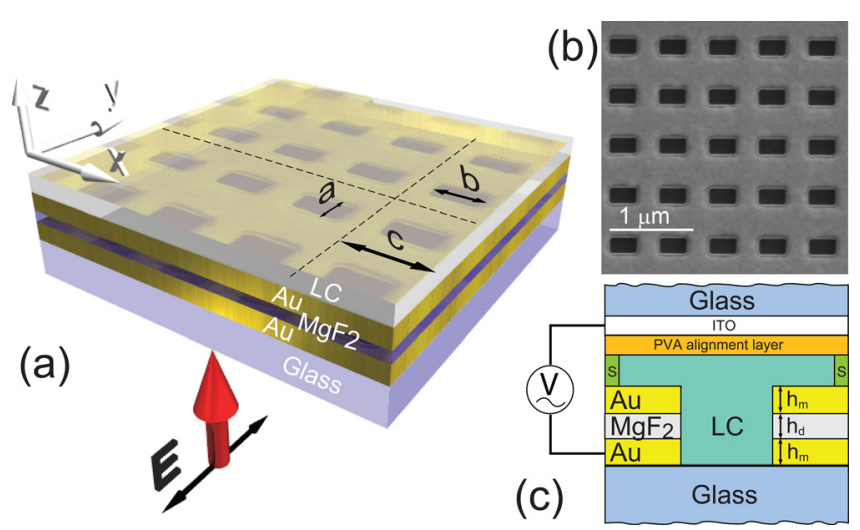

FIG. 1. (Color online) (a) Schematic of the LC infiltrated fishnet metamaterial. (b) Scanning electron microscope image (top view) of the fabricated fishnet metamaterials. (c) Side view of the LC cell: DL is a dielectric layer, $\mathrm{S}-100 \mu \mathrm{m}$ thick plastic spacers. Parameters for the fabricated $\mathrm{Au}-\mathrm{MgF}_{2}-\mathrm{Au}$ fishnet are $h_{d}=h_{m}=50 \mathrm{~nm}, a=190 \mathrm{~nm}, b=350 \mathrm{~nm}$, and $c=600 \mathrm{~nm}$.

First, we measure the linear transmission properties of the fabricated fishnets using a linearly polarized white halogen tungsten light as shown in Fig. 2(a). We focus the image of the aperture onto the metamaterial sample and collect the transmitted light in the infra-red spectral region onto an optical spectrum analyzer (OSA). Fig. 2(b) shows the linear transmission spectrum of the fishnet before [Figs. 2a(b-i)] and after infiltration [Figs. 2a(b-ii)]. The solid curves correspond to our experimental measurements, while the dashed curves are based on finite-difference time-domain numerical simulations. In our simulations, we use six-term Drude-Lorentz expression for the dielectric permittivity of gold ${ }^{25}$ and a constant refractive index of 1.38 for the $\mathrm{MgF}_{2}$. While there is a factor of two difference in the magnitude of transmission

(a)
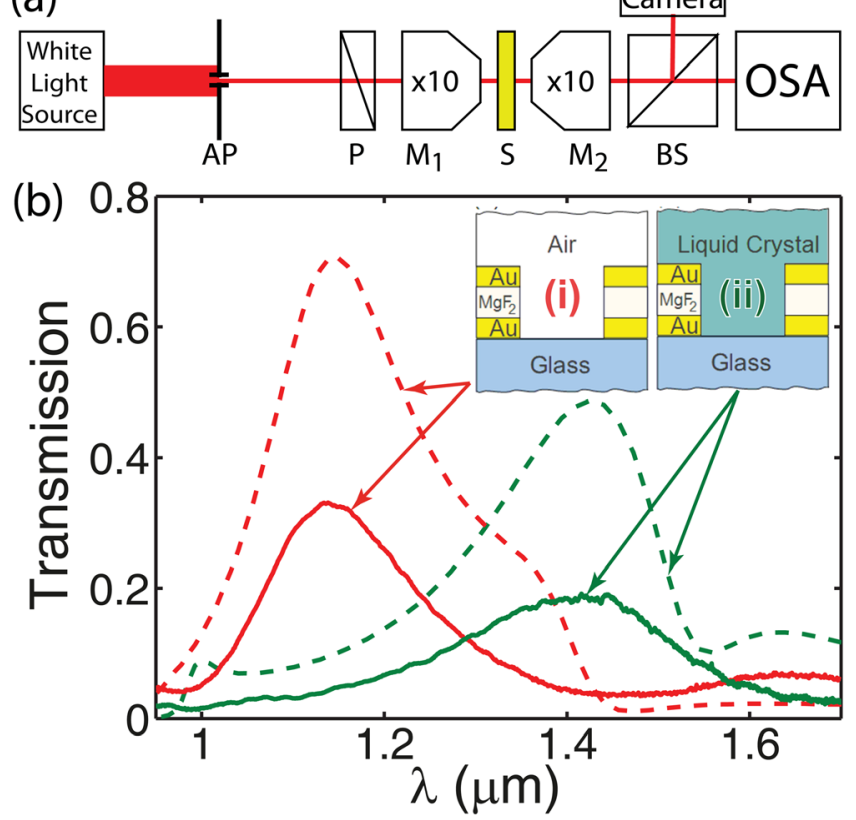

FIG. 2. (Color online) (a) Experimental setup for the transmission spectral measurement of the fishnet metamaterial, where AP is an aperture, BSbeam splitter, $\mathrm{S}$ - fishnet sample, $\mathrm{P}$ - polarizer, OSA — optical spectrum analyzer, and $\mathrm{M}_{1}, \mathrm{M}_{2}$ - microscope objectives. (b) Measurement of linear transmission of the LC cell, including $\mathrm{Au}-\mathrm{MgF}_{2}-\mathrm{Au}$ fishnet metamaterials. Solid lines - experimental results; dashed lines_-numerical simulations. (i) empty structure; (ii) the structure fully infiltrated with E7 nematic liquid crystal. between our measurements and the numerical calculations, there is good correspondence in the wavelength positions of the spectral maxima. We attribute the lower transmission seen in experiment to the inevitable roughness of the film and the higher losses in the sputtered gold. More importantly, from Fig. 2(b), we can conclude that a complete LC infiltration of the nanoscale holes of the fishnet structure has been achieved. If air pockets were formed during the infiltration process, the transmission spectrum would show a peak lying in between the peaks of both curves (i) and (ii), as confirmed by numerical simulations.

The next important point of our experiments is the investigation of alignment of the LC molecules. The use of a PVA layer on the top cover-glass slide forming our LC cell ensures that near this layer, the LC molecules are aligned parallel to the surface. However, it is unknown what the alignment of the LC molecules at the fishnet surface is (in particular inside the holes) and how the top alignment propagates inside the $100 \mu \mathrm{m}$ thick LC cell. Importantly, for a strong nonlinear response of the $\mathrm{LC}$, it is preferable that the LC molecules are aligned perpendicular (or with a small angle) to incident light electric field. Therefore, if the top alignment is fully preserved, a bias electric field across the top and bottom electrodes of the LC cell will need to be applied to ensure proper molecular orientation of the LC molecules. However, our experimental measurements of the transmission spectra with and without applied bias electric field show negligible change in transmission. This indicates that the LC molecules are aligned vertically inside the holes of the fishnet. This is again confirmed with numerical simulations showing that for LC reorientation from horizontal to vertical, the transmission maximum will experience a spectral shift of the order of $100 \mathrm{~nm} .^{22}$ The observed fixed vertical alignment is possible due to surface anchoring of the LC molecules to the side walls of the fishnet structure. We note that this vertical alignment of the LC molecules inside the holes favors the re-orientational nonlinearity of the LC through illumination with $y$-polarized optical fields [see Fig. 1(a)].

In order to study the dependence of optical transmission on light intensity, we illuminate the infiltrated metamaterials with a laser beam from a telecom fiber laser (Pritel) at $1550 \mathrm{~nm}$, which is mildly focused to a $20 \mu \mathrm{m}$ size spot. The experimental setup is depicted in Fig. 3(a). The fiber laser operates in a pulsed regime with a repetition rate of $5 \mathrm{MHz}$ and spectral bandwidth of $0.3 \mathrm{~nm}$. As such, the laser beam acts as a quasi-cw illumination, because the typical time response of the LC is of the order is $1 \mathrm{~ms}$. The fishnet structure is excited through the substrate side in order to minimize any effects from the bulk ( $100 \mu \mathrm{m}$ thick) layer of LC. This is in contrast to the recent experiments of LC metamaterials ${ }^{26}$ where the polarization rotation in the bulk part of the LC layer is responsible for the change of light transmission. The transmitted power in our experiment is detected by a polarization independent photo-detector, however depolarization effects through the structure were found to be negligible. The particular excitation laser wavelength is positioned on the long-wavelength side of the transmission maxima [see Fig. 2a(b-ii)] and near the wavelength of the gap plasmon resonance, thus operating in the expected negative index spectral region. 
(a)
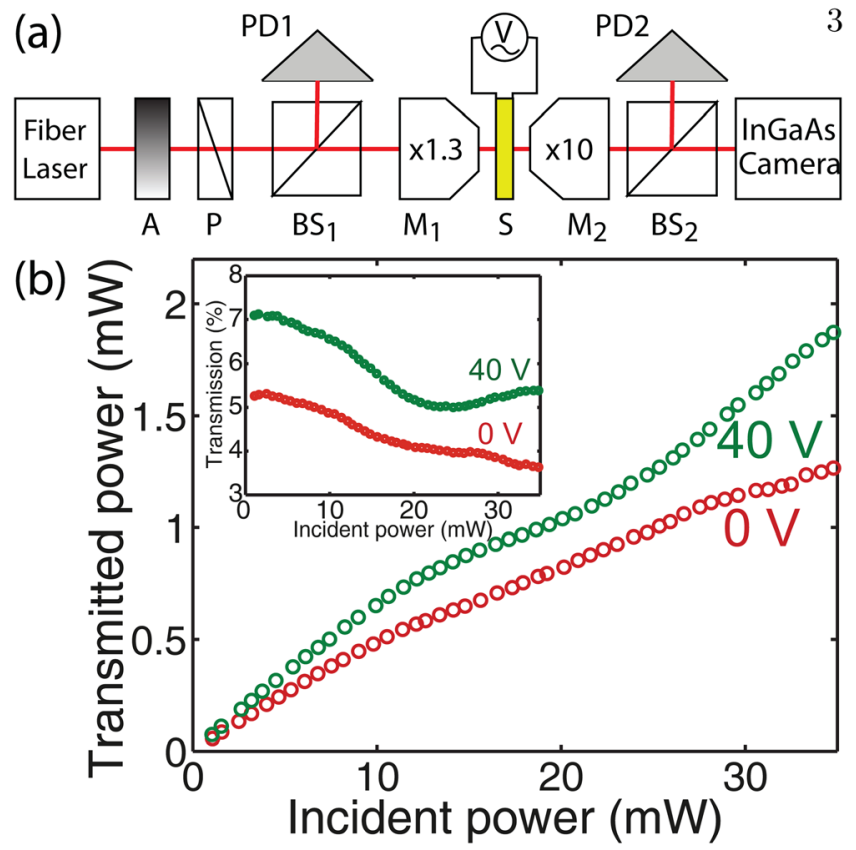

FIG. 3. (Color online) (a) Experimental setup for nonlinear transmissions measurement at $1550 \mathrm{~nm}$. A-attenuator, $\mathrm{PD}_{1}, \mathrm{PD}_{2}$ - photodiode detectors, $\mathrm{P}$-polarizer, $\mathrm{BS}_{1}, \mathrm{BS}_{2}$ - beam splitters, and $\mathrm{M}_{1}, \mathrm{M}_{2}$-microscope objectives. (b) Measured transmission at $1550 \mathrm{~nm}$ vs. input power for two bias voltages $0 \mathrm{~V}$ and $40 \mathrm{~V}$. Dashed line-linear dependence. Inset: Normalized transmission vs. incident laser power.

First, we perform a reference measurement by testing the transmission of the entire stack of layers in the LC cell, but without the fishnet metamaterial and obtain a perfectly linear dependence of the transmission with power. The linear dependence is well preserved regardless of the applied bias electric field, again showing that bulk nonlinear effects in the LC cell are negligible. However, the dependence of transmission on laser power dramatically changes if the fishnet metamaterial is placed into the laser beam (Fig. 3). In this case, we observe a drop (sub-linear dependence) of the transmitted power in comparison to the linear dependence at low light intensities. For completeness, Fig. 3(b-inset) also shows the normalized transmission with an increase of the incident laser power. At low input powers, the transmission through the infiltrated structure is $\sim 5 \%$ for zero applied bias field but with the increase of the laser power a differential drop of transmission of approximately $30 \%$ is observed. The dependency of the transmission is measured for the power range from zero to $35 \mathrm{~mW}$. Above these power levels, the thermal effects start to dominate the transmission, which is reflected in an increased noise and non-stationary dynamics.

We also observe that the measured transmission drop is strongly dependent on the application of a bias electric field, confirming the strong molecular re-orientation of the LCs inside the holes of the fishnet metamaterials. For a bias voltage of $40 \mathrm{~V}$ [Fig. 3(b)], the linear transmission is slightly increased due to the change of LC domain walls in the bulk part of the LC cell, however again there is a drop in the transmission with increase of incident laser power. As seen in Fig. 3(b-inset), the drop in transmission saturates at about $20 \mathrm{~mW}$ and a differential change of $30 \%$ is observed. This non-monotonic dependence is an indication of strong inter- play between optical and electrical reorientation of the LC molecules at these laser powers. This interplay demonstrates an important mechanism of electrically controlled optical nonlinearity in our fishnet metamaterials structures. We note that the exact interplay depends strongly on the mechanisms of surface anchoring of the LC molecules in the nano-holes and further studies are required to understand these effects.

Our results to alter the transmission and correspondingly the refractive index of fishnet metamaterial all-optically by the light itself represent an important milestone in the development of metamaterials with enhanced nonlinear response. The possible implementation of metamaterials in signal processing devices further requires the exploration of ultrafast nonlinear optics in metamaterials. While recent pumpprobe experiments in fishnets ${ }^{27}$ are a step forward in this direction, the self-action of ultra-short light signals still remains to be tested.

In conclusion, we have fabricated and studied experimentally fishnet metamaterial structures with strong optical nonlinearity due to their infiltration with nematic liquid crystals. We have observed a large change in the optical transmission through the structure at moderate laser powers of just a few milliwatts due to orientational nonlinearity of the liquid crystal. Importantly, we have also shown the possibility of controlling the nonlinear response of the fishnet metamaterials by application of a bias electric field, thus opening further opportunities for the realization of optical materials with electrically tunable nonlinear response.

We acknowledge the support by the Australian Research Council, the Australian National Computational Infrastructure, and the ACT Node of Australian National Fabrication Facility. We thank A. Zayats, A. Miroshnichenko, and E. Brasselet for the useful discussions.

${ }^{1}$ G. A. Wurtz and A. V. Zayats, Laser Photon. Rev. 2, 125 (2008).

${ }^{2}$ S. Carretero-Palacios, A. Minovich, D. N. Neshev, Yu. S. Kivshar, F. J. Garcia-Vidal, L. Martin-Moreno, and S. G. Rodrigo, Opt. Lett. 35, 4211 (2010).

${ }^{3}$ J. B. Pendry, A. J. Holden, D. J. Robbins, and W. J. Stewart, IEEE Trans. Microwave Theory Tech. 47, 2075 (1999).

${ }^{4}$ A. A. Zharov, I. V. Shadrivov, and Yu. S. Kivshar, Phys. Rev. Lett. 91, 037401 (2003).

${ }^{5}$ B. Wang, J. Zhou, T. Koschny, and C. M. Soukoulis, Opt. Express 16, 16058 (2008).

${ }^{6}$ D. Huang, E. Poutrina, and D. R. Smith, Appl. Phys. Lett. 96, 104104 (2010).

${ }^{7}$ N. I. Zheludev, Science 328, 582 (2010).

${ }^{8}$ A. D. Boardman, V. V. Grimalsky, Y. S. Kivshar, S. V. Koshevaya, M. Lapine, N. M. Litchinitser, V. N. Malnev, M. Noginov, Y. G. Rapoport, and V. M. Shalaev, Laser Photon. Rev. 5, 287 (2011).

${ }^{9}$ A. I. Maimistov and I. R. Gabitov, Eur. Phys. J. 147, 265 (2007).

${ }^{10}$ N. M. Litchinitser and V. M. Shalaev, Laser Phys. Lett. 5, 411 (2008).

${ }^{11}$ M. W. Klein, C. Enkrich, M. Wegener, and S. Linden, Science 313, 502 (2006).

${ }^{12}$ E. Kim, F. Wang, W. Wu, Z. Yu, and Y. R. Shen, Phys. Rev. B 78, 113102 (2008).

${ }^{13}$ M. J. Huttunen, G. Bautista, M. Decker, S. Linden, M. Wegener, and M. Kauranen, Opt. Mater. Express 1, 46 (2011).

${ }^{14}$ A. E. Nikolaenko, F. De Angelis, S. A. Boden, N. Papasimakis, P. Ashburn, E. Di Fabrizio, and N. I. Zheludev, Phys. Rev. Lett. 104, 153902 (2010).

${ }^{15}$ G. A. Wurtz, R. Pollard, W. Hendren, G. P. Wiederrecht, D. J. Gosztola, V. A. Podolskiy, and A. V. Zayats, Nat. Nano 6, 107 (2011).

${ }^{16}$ I. C. Khoo, J. Opt. Soc. Am. B 28, A45 (2011). 
${ }^{17}$ B. Wang, M. A. Dndar, A. Y. Silov, R. Nötzel, F. Karouta, S. He, and R. W. van der Heijden, Opt. Lett. 35, 2603 (2010).

${ }^{18}$ P. R. Evans, G. A. Wurtz, W. R. Hendren, R. Atkinson, W. Dickson, A. V. Zayats, and R. J. Pollard, Appl. Phys. Lett. 91, 043101 (2007).

${ }^{19}$ W. Dickson, G. A. Wurtz, P. R. Evans, R. J. Pollard, and A. V. Zayats, Nano Lett. 8, 281 (2007).

${ }^{20}$ I. C. Khoo, D. H. Werner, X. Liang, A. Diaz, and B. Weiner, Opt. Lett. 31, 2592 (2006).

${ }^{21}$ X. Wang, D.-H. Kwon, D. H. Werner, I. C. Khoo, A. V. Kildishev, and V. M. Shalaev, Appl. Phys. Lett. 91, 143122 (2007).

${ }^{22}$ A. Minovich, D. N. Neshev, D. A. Powell, I. V. Shadrivov, and Yu. S. Kivshar, Appl. Phys. Lett. 96, 193103 (2010).
${ }^{23}$ S. Xiao, U. K. Chettiar, A. V. Kilkdishev, V. Drachev, I. C. Khoo, and A. V. Shalaev, Appl. Phys. Lett. 95, 033115 (2009).

${ }^{24}$ A. Minovich, D. N. Neshev, D. A. Powell, I. V. Shadrivov, M. Lapine, H. T. Hattori, H. H. Tan, C. Jagadish, and Yu. S. Kivshar, Phys. Rev. B 81, 115109 (2010).

${ }^{25}$ A. D. Rakic, A. B. Djurisic, J. M. Elazar, and M. L. Majewski, Appl. Opt. 37, 5271 (1998).

${ }^{26}$ B. Kang, J. H. Woo, E. Choi, H.-H. Lee, E. S. Kim, J. Kim, T.-J. Hwang, Y.-S. Park, D. H. Kim, and J. W. Wu, Opt. Express 18, 16492 (2010).

${ }^{27}$ K. M. Dani, Z. Ku, P. C. Upadhya, R. P. Prasankumar, A. J. Taylor, and S. R. J. Brueck, Opt. Express 19, 3973 (2011). 